\title{
Laboratory study of astrophysical collisionless shock at SG-II laser facility
}

Dawei Yuan ${ }^{1}$, Huigang Wei ${ }^{1}$, Guiyun Liang ${ }^{1}$, Feilu Wang ${ }^{1}$, Yutong Li ${ }^{2,5}, 11$, Zhe Zhang ${ }^{2}$, Baojun Zhu ${ }^{2}$, Jiarui Zhao ${ }^{2}$, Weiman Jiang ${ }^{2}$, Bo $\mathrm{Han}^{3}$, Xiaoxia Yuan ${ }^{3}$, Jiayong Zhong ${ }^{3,5}$, Xiaohui Yuan ${ }^{4}$, Changbo $\mathrm{Fu}^{6}$, Xiaopeng Zhang ${ }^{6}$, Chen Wang ${ }^{7}$, Guo Jia ${ }^{7}$, Jun Xiong ${ }^{7}$, Zhiheng Fang ${ }^{7}$, Shaoen Jiang ${ }^{8}$, Kai Du ${ }^{8}$, Yongkun Ding ${ }^{8}$, Neng Hua ${ }^{9}$, Zhanfeng Qiao ${ }^{9}$, Shenlei Zhou ${ }^{9}$, Baoqiang Zhu ${ }^{9}$, Jianqiang Zhu', Gang Zhao ${ }^{1,10}$, and Jie Zhang ${ }^{4,5}$

${ }^{1}$ Key Laboratory of Optical Astronomy, National Astronomical Observatories, Chinese Academy of Sciences, Beijing 100012, China

${ }^{2}$ National Laboratory for Condensed Matter Physics, Institute of Physics, Chinese Academy of Sciences, Beijing 100190, China

${ }^{3}$ Department of Astronomy, Beijing Normal University, Beijing 100875, China

${ }^{4}$ Key Laboratory for Laser Plasmas (MoE) and Department of Physics and Astronomy, Shanghai Jiao Tong University, Shanghai 200240, China

${ }^{5}$ Collaborative Innovation Center of IFSA (CICIFSA), Shanghai Jiao Tong University, Shanghai 200240, China

${ }^{6}$ INPAC and School of Physics and Astronomy, Shanghai Jiao Tong University, Shanghai Key Laboratory for Particle Physics and Cosmology, Shanghai 200240, China

${ }^{7}$ Shanghai Institute of Laser Plasma, Shanghai 201800, China

${ }^{8}$ Research Center for Laser Fusion, China Academy of Engineering Physics, Mianyang 621900, China

${ }^{9}$ National Laboratory on High Power Laser and Physics, Chinese Academy of Sciences, Shanghai 201800, China

${ }^{10}$ School of Astronomy and Space Science, University of Chinese Academy of Sciences, Beijing 101408, China

${ }^{11}$ School of Physical Sciences, University of Chinese Academy of Sciences, Beijing 100049, China

(Received 26 November 2017; revised 19 March 2018; accepted 21 June 2018)

\begin{abstract}
Astrophysical collisionless shocks are amazing phenomena in space and astrophysical plasmas, where supersonic flows generate electromagnetic fields through instabilities and particles can be accelerated to high energy cosmic rays. Until now, understanding these micro-processes is still a challenge despite rich astrophysical observation data have been obtained. Laboratory astrophysics, a new route to study the astrophysics, allows us to investigate them at similar extreme physical conditions in laboratory. Here we will review the recent progress of the collisionless shock experiments performed at SG-II laser facility in China. The evolution of the electrostatic shocks and Weibel-type/filamentation instabilities are observed. Inspired by the configurations of the counter-streaming plasma flows, we also carry out a novel plasma collider to generate energetic neutrons relevant to the astrophysical nuclear reactions.
\end{abstract}

Keywords: collisionless shock; electromagnetic field; high power lasers; laboratory astrophysics

\section{Introduction}

High power lasers can create extreme conditions in the laboratory relevant to astrophysical systems ${ }^{[1-3]}$. With the

Correspondence to: Y. Li, Beijing National Laboratory of Condensed Matter Physics, Institute of Physics, Chinese Academy of Sciences, Beijing 100190, China; G. Zhao, Key Laboratory of Optical Astronomy, National Astronomical Observatories, Chinese Academy of Sciences, Beijing 100012, China; J. Zhang, Key Laboratory for Laser Plasmas (MoE) and Department of Physics and Astronomy, Shanghai Jiao Tong University, Shanghai 200240, China. Email: ytli@iphy.ac.cn (Y. Li); gzhao@bao.ac.cn (G. Zhao); jzhang1@ @ sjtu.edu.cn (J. Zhang). scaling law ${ }^{[4]}$, it presents a new route, laboratory astrophysics, to investigate the astrophysical scenarios, such as shock generation in supernova explosions ${ }^{[5]}$, magnetic reconnection occurring at solar flares ${ }^{[6,7]}$, and Herbig-Haro objects associated with young stellar object formation ${ }^{[8]}$.

One of the hottest research fields is astrophysical shocks, which are ubiquitous and observed in a wide range of astrophysical environments, such as solar-terrestrial space, supernova explosions and gamma-ray bursts. Figure 1(a) shows a typical composite image of the supernova remnant 1006 (SNR 1006) caused by the destruction of a white 
dwarf star, which is located about 7000 light years from the Earth ${ }^{[9]}$. A sharp edge appears in the interaction region where the exploded matters with high velocity penetrate through the rare interstellar mediums (ISMs), indicating that a shock is formed. The shock transition region is measured about $0.04 \mathrm{pc}\left(=1.2 \times 10^{17} \mathrm{~cm}\right), 1 / 400$ of the mean free path (MFP $\sim 13 \mathrm{pc})^{[10]}$. Obviously, it is collisionless and mediated by the collective effect, instead of the Coulomb collisions. This shock has attracted much interest in its formation mechanism, generation/amplification of the magnetic fields ${ }^{[11,12]}$, and the spray of the energetic cosmic rays $^{[13-15]}$.

Figure 1(b) shows a schematic drawing of the counterstreaming plasma flows (CPFs) in laboratory, which are designed to study the shock. Both flows are generated from the opposing target foils. Two schemes (Cases I and II) are used to generate CPFs: (Case I) one is using two laser bunches directly ablating the facing surfaces of the foils; and (Case II) the other one is only using one laser bunch ablating a foil to blow out an incoming flow, the reverse flow is generated due to photoionization of the other foil by the intense X-ray from the laser-irradiation target.

No matter which scheme is applied, it must achieve the collisionless conditions between CPFs, i.e., the MFP larger than the interaction scale, $L$ (target separation or shock transition width, in our experiment $L=4.5 \mathrm{~mm}$ ). The simplified expression of MFP can be written in Gaussian units as ${ }^{[16]} \lambda_{m f p}=m_{i}^{2} v_{12}^{4} /\left(4 \pi e^{4} Z^{4} n_{i} \ln \Lambda_{12}\right)$, where $m_{i}$ is the ion mass, $v_{12}=2 v$ is the relative velocity of each flow, $e$ is the electric charge, $Z$ is the average charge state, $n_{e}=Z n_{i}$ is the electron density and $\ln \Lambda$ is the Coulomb logarithm. We can see that the desirable collisionless conditions in the laboratory are higher flow velocity and lower- $Z$ target material. We can increase the driven laser energy, $E_{L}$, or optimize the laser absorption coefficient, $f$, to increase the flow velocity, which mainly depends on the absorbed driven

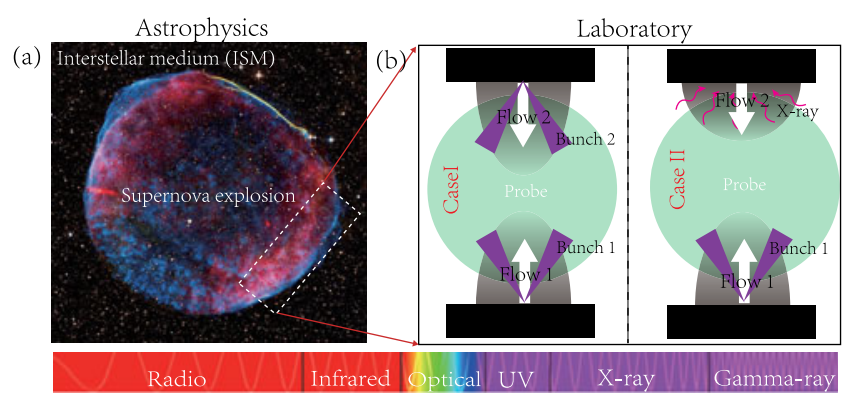

Figure 1. (a) A composite image of collisionless shock formed in the SNRs ${ }^{[18]}$. Color stands for the observation wavelength, as shown in the color bar. (b) The experimental configurations to simulate the astrophysical CPFs. Here, two schemes are used to generate counter-streaming flows. Case $\mathrm{I}$ is a symmetrical one, where both flows are directly ablating both facing surfaces of the foils. Case II is an unsymmetrical one, where only one foil is ablating by one bunch and the other side foil is heated by the $\mathrm{X}$ ray from laser-target interaction. The probe beam (outwards) transversely passes through the interaction region for optical diagnostics. laser energy $\left(1 / 2 n_{i} m_{i} v^{2} \times V=E_{k}=f E_{L}\right.$, where $n_{i}$ is the ion number density and $V$ is the plasma volume $)^{[17]}$. The lower- $Z$ material of the solid target can be chosen as $\mathrm{CH}$, $\mathrm{CD}, \mathrm{DLi}$, etc.

Here we will review the achievements of the collisionless shock at SG-II laser facility ${ }^{[19-21]}$, which can deliver a total energy of $2.0 \mathrm{~kJ}$ in $1 \mathrm{~ns}$ at $3 \omega(351 \mathrm{~nm})$. Eight SGII laser beams are divided into two bunches, each bunch consisting of four beams. The overlapped focal spot is $150-200 \mu \mathrm{m}$, giving an intensity of $\sim 10^{15} \mathrm{~W} \cdot \mathrm{cm}^{-2}$. The main diagnostics is using optical probe, including Nomarski interferometer and shadowgraphy. As shown in Figure 1(b), a probe beam with a wavelength of $2 \omega(527 \mathrm{~nm})$ and duration of $30 \mathrm{ps}$ transversely passes through the interaction region, measuring the density, flow velocity and so on. In this paper, the evolution of the electrostatic shock (ES) and filamentation instability is successfully observed by optimizing experimental conditions in CPFs. Inspired by the configuration of the CPFs, we also perform an exploratory experiment relevant to neutron astrophysics to distinguish between collisionless and collisional effects in CPFs.

\section{Experimental results}

\subsection{The evolution of the symmetrical CPFs}

The left panel in Figure 2 shows the typical interferograms of the evolution of the $\mathrm{CPFs}$, which are produced by two laser-bunches ablating $\mathrm{CH}$ foils ${ }^{[22-24]}$. The right panel shows the corresponding density profile obtained by the Abel inversion. Before shock formation $(t<2 \mathrm{~ns})$, we measure the free expansion of the plasma flow with electron density of $10^{19} \mathrm{~cm}^{-3}$ and flow velocity of $1000 \mathrm{~km} \cdot \mathrm{s}^{-1}$. During shock formation $(t \sim 2 \mathrm{~ns})$, both flows interact with each other and result in the sudden fringes shift at the midplane, indicating that a large-density jump $\left(n_{\text {shock }} / n_{\text {flow }} \sim 3\right)$ is generated. After that $(t>3 \mathrm{~ns})$, the shifted fringes become smooth and filament structures parallel to the flow direction appear in the central region, indicating that the excited shock is perhaps dissipated by the growing filamentary structures $(t>5 \mathrm{~ns})$. Since the MFP $\left(\lambda_{i i}\right)$ scales as $1 / Z^{4}$, the $\lambda_{i i}$ for hydrogen ions $(Z=1)$ will be higher than that for carbon ions $(Z=6)$. Here we just use $Z=6$ to calculate the lower limit of MFP, whose value is estimated as $180 \mathrm{~mm}$, indicating that ions from inter-flows can freely interpenetrate each other. Consequently, these observed features in the CPFs should be induced by plasma collective behaviors, instead of the hydrodynamic stagnation.

Relevant works ${ }^{[25,26]}$ show that two candidate instabilities can be self-generated under such experimental conditions $\left(n_{e} \sim 10^{19} \mathrm{~cm}^{-3}, v \sim 10^{8} \mathrm{~cm} \cdot \mathrm{s}^{-1}, T_{e} \sim 0.2-1 \mathrm{keV}\right)$. One is electrostatic instability ${ }^{[27]}$, and the other one is Weibeltype/filamentation instability ${ }^{[28,29]}$. Simulation results show that both instabilities are competitive. The filamentation 

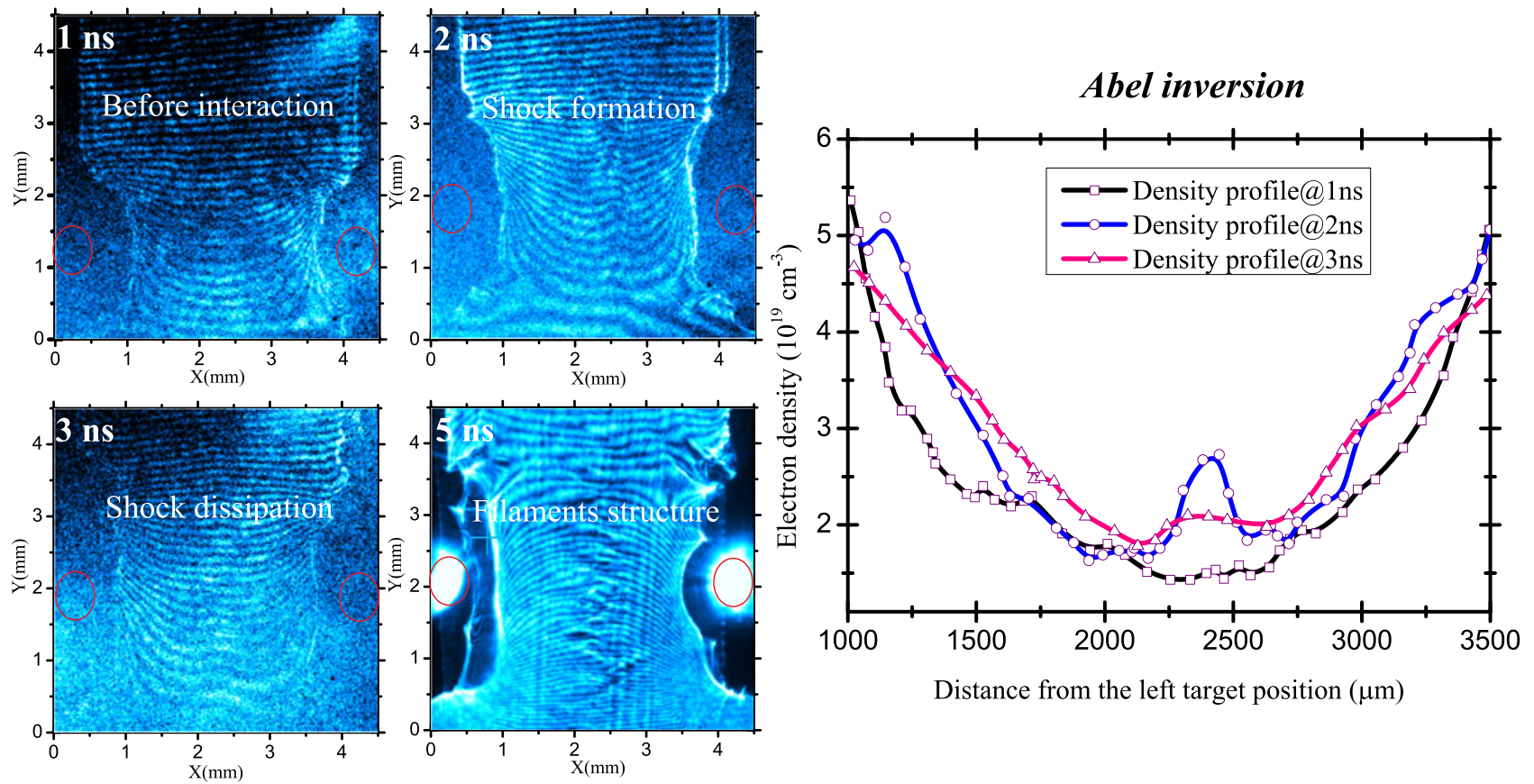

Figure 2. The evolution of the counter-streaming flows obtained by a Nomarski interferometer. The red circle in the raw images stands for the laser focal spot. When both flows coming from the opposing foils interpenetrate each other at the midplane at 2 ns shown in Abel inversion image, the plasma density increases by the unanticipated factor of 3 (from $1 \times 10^{19} \mathrm{~cm}^{-3}$ to $2.8 \times 10^{19} \mathrm{~cm}^{-3}$ ), indicating that a shock has been generated. The width is measured as about $300 \mu \mathrm{m}$, much smaller than the MFPs. Obviously, it is collisionless. Subsequently $(\sim 3-5 \mathrm{~ns})$, the collisionless shock is dissipated by the growing filamentation structures.

instability developed later would destroy the evolution of the electrostatic instability ${ }^{[26]}$. This evolution is consistent with the observed experimental results. In order to measure the whole process of the ES, we should suppress the growth of the Weibel-type instability. According to the growth rate of the Weibel-type instability,

$$
\gamma \propto \frac{v}{c} \sqrt{\frac{4 \pi n_{i} Z^{2} e^{2}}{m_{i}}} \propto \frac{Z e}{A c m_{p}} \sqrt{8 \pi f E_{L}},
$$

we have two methods to adjust the Weibel-type instability. One is symmetrical case (Case I), replacing the target material, i.e., changing the value, $(Z / A) \sqrt{f}$. The other one is unsymmetrical case (Case II), changing the driven laser energy $E_{L}$. Here we define the symmetrical case and unsymmetrical case according to the driven laser conditions instead of the plasma flow conditions. The symmetrical case stands for two bunches heating the target foils, and the unsymmetrical case stands for only one bunch heating the target foil.

\subsection{Collisionless electrostatic shock formation and evolution in the CPFs}

\subsubsection{Unsymmetrical case}

Figure 3 shows the ES in the CPFs at $5 \mathrm{~ns}$ and $9 \mathrm{~ns}^{[30]}$. In this experiment configuration, the laser-driven high-velocity, low-density left flow interacts with the low-velocity, highdensity flow generated by X-ray ionization at the surface of the right foil. Both flow densities are measured by the interferograms, i.e., $n_{e}^{\text {Left }} \sim 2 \times 10^{18} \mathrm{~cm}^{-3}$ and $n_{e}^{\text {Right }} \sim 6 \times$ $10^{18} \mathrm{~cm}^{-3}$. Some structures appear near to $x=3.6-3.7 \mathrm{~mm}$ at $5 \mathrm{~ns}$, indicating that both flow velocities can be estimated as $v^{\text {Left }} \sim 830 \mathrm{~km} \cdot \mathrm{s}^{-1}$ and $v^{\text {Right }} \sim 230 \mathrm{~km} \cdot \mathrm{s}^{-1}$. The corresponding MFPs are calculated as $25-35 \mathrm{~mm}$, confirming that both flows interaction is collisionless. These observed structures at $5 \mathrm{~ns}$ in Figures 3(a) and 3(c) are identified as a large-density jump. However, its value is difficult to measure because of such a larger density gradient blocking the probe propagation. Anyway, such a large-density jump indicates that a collisionless shock has been generated at $5 \mathrm{~ns}$. With the development of the shock, it continues to move from $x=3.6-3.7 \mathrm{~mm}$ at $5 \mathrm{~ns}$ to $x=3.1 \mathrm{~mm}$ at $9 \mathrm{~ns}$, i.e., it propagates with a velocity of $v_{s} \sim 125-150 \mathrm{~km} \cdot \mathrm{s}^{-1}$. The corresponding Mach number is about 9-11, suggesting a strong collisionless shock formation. The typical features of the shock at 9 ns are shown in Figures 3(e) and 3(f), and the density jump decreases to $n_{\text {shock }} / n_{\text {flow }} \sim 3.89 \pm 0.85$ with the whole transition region of about $200 \mu \mathrm{m}$.

To clarify the generation mechanism of the shock, a quasi-one-dimensional particle-in-cell (PIC) simulation is performed under the same experimental conditions. From Figure 4 in Ref. [30], a self-generated bipolar electrostatic field mediated shock formation appears at $x / \lambda_{e} \sim 26$ in the beginning, and then propagates toward $\pm x$ directions. 

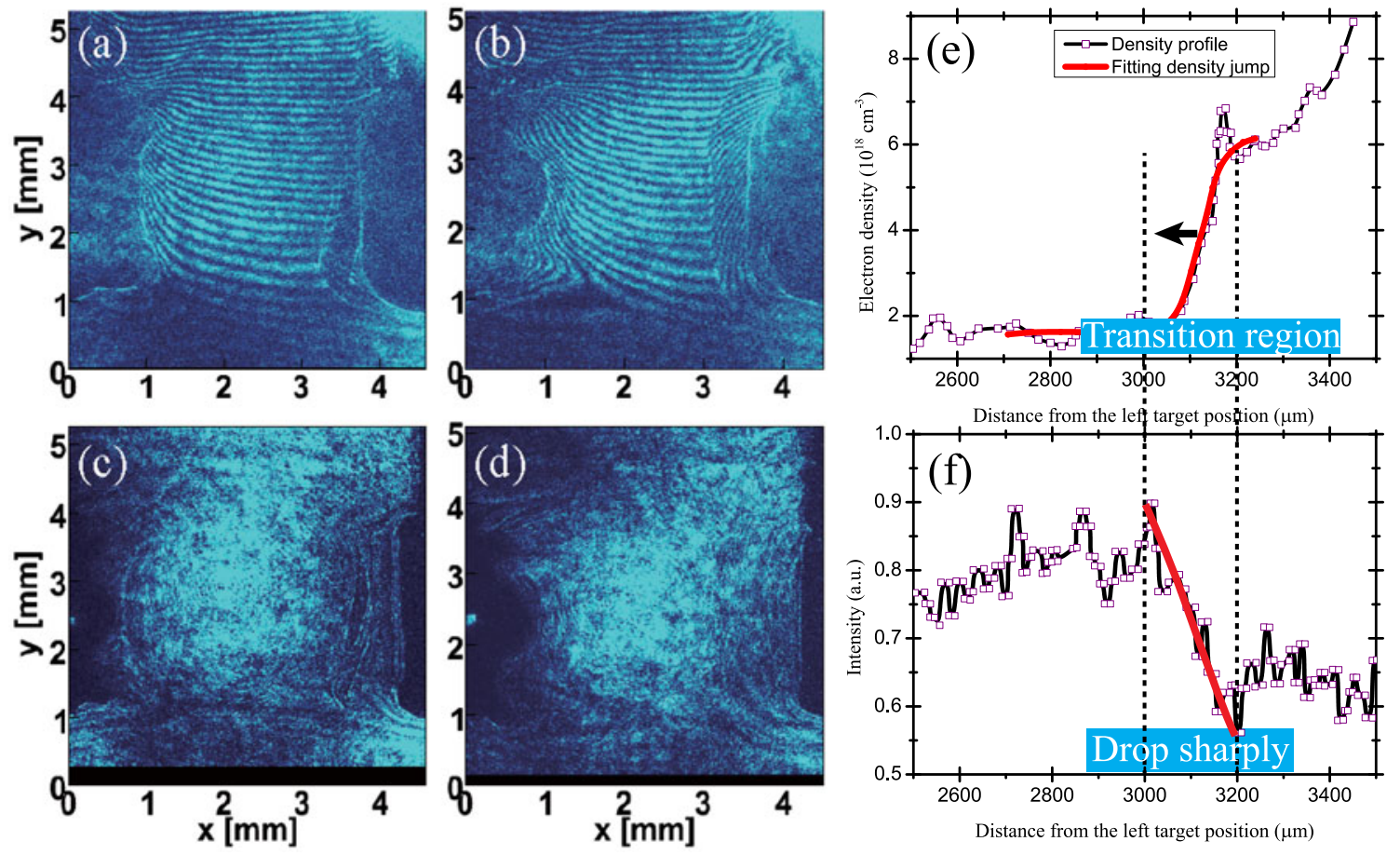

Figure 3. Collisionless shock formation and evolution in unsymmetrical $\mathrm{CPFs}^{[30]}$. (a) and (b) show the interferogram obtained at $5 \mathrm{~ns}$ and $9 \mathrm{~ns}$, respectively. (c) and (d) are the corresponding shadowgraphs of (a) and (b). (e) and (f) show the density profile and the intensity profile at $9 \mathrm{~ns}$.

The incoming ions will be slowed down or reflected back into upstream. Consequently, ions are accumulated into the interaction region until the density jump conditions are fulfilled ${ }^{[30,31]}$.

\subsubsection{Symmetrical case}

Figure 4 shows the ES formation and evolution in the CPFs at $6 \mathrm{~ns}$ and $10 \mathrm{~ns}$. The $\mathrm{CH}$ target material is replaced by the $\mathrm{Cu}$. In this experiment configuration, two similar laserproduced plasma flows interact with each other $\left(n_{e} \sim 1 \times\right.$ $\left.10^{19} \mathrm{~cm}^{-3}, 2 v \sim 7.5 \times 10^{7} \mathrm{~cm} \cdot \mathrm{s}^{-1}\right)$. Two overlapped shocks are formed in the central region and then evolve into two separated shocks from the downstream to upstream. The MFP $(16-500 \mathrm{~mm})$ is also much larger than the shock width $(400-700 \mu \mathrm{m})$. According to the heuristic model proposed by Park et al. in Ref. [32], the width of the ES transition region can be estimated as $L_{E S}=K \frac{v}{\omega_{p i}} \frac{W}{T_{e}} \approx$ (600-800) $\mu \mathrm{m}$, in agreement with our measurement $(L \sim$ 450-800 $\mu \mathrm{m}$ ). However, the width of the Weibel-type shock should be estimated as $L_{E M}=K \frac{v}{\omega_{p i}} \approx 15 \mathrm{~mm}$, much larger than our target separation. The theoretical analysis shows that the observed shocks should be electrostatic. The corresponding PIC simulations also confirm our understanding. As shown in Figure 5 in Ref. [20], a strong bipolar electrostatic field resulted from the instability excites the shocks. Large temperature and density gradients between upstream and downstream drive the shock from downstream to upstream.

\subsection{Weibel/filamentation instability in the symmetrical case}

Weibel instability is a promising candidate for creating astrophysical shocks. It is a typical electromagnetic phenomenon, driven by the plasma anisotropy. Under the current laser-plasma conditions, i.e., the electron thermal velocity is larger than the flow velocity and the ion thermal velocity is smaller than the flow velocity, the ions freely interpenetrate each other in the presence of a single thermalized electron background. Therefore, it is called ion-ion driven Weibeltype instability. The signature of Weibel instability is that the filamentary structures form and extend in the axis of flow direction. The self-generated magnetic field grows from linear phase to nonlinear phase until saturation. Although many groups ${ }^{[29,33-35]}$ have been devoted into investigation of the Weibel-mediated shock formation, the required exact physical conditions for such an occurrence are still unclear.

According to Equation (1), we can find that increasing the flow velocity and density can enhance the growth rate of the Weibel instability. Here we demonstrate the Weibel instability in $\mathrm{CPFs}$ generated by $\mathrm{CH}-\mathrm{Al}$ foils. Figure 5 shows the typical results of evolution of the Weibel-type instability. The left flow (in the interferogram) is generated by one bunch ablation of $\mathrm{CH}$ foil, and the right flow is generated by the other bunch ablation of Al foil. Such a configuration can produce a relatively high density $\left(n_{e} \sim\right.$ $\left.3 \times 10^{19} \mathrm{~cm}^{-3}\right)$ and similar velocity $\left(v \sim 0.8 \times 10^{8} \mathrm{~cm} \cdot \mathrm{s}^{-1}\right)$ plasma flows, in comparison with $\mathrm{CH}-\mathrm{CH}$ case in Figure 2 $\left(n_{e} \sim 1 \times 10^{19} \mathrm{~cm}^{-3}, v \sim 1 \times 10^{8} \mathrm{~cm} \cdot \mathrm{s}^{-1}\right)$. The typical 

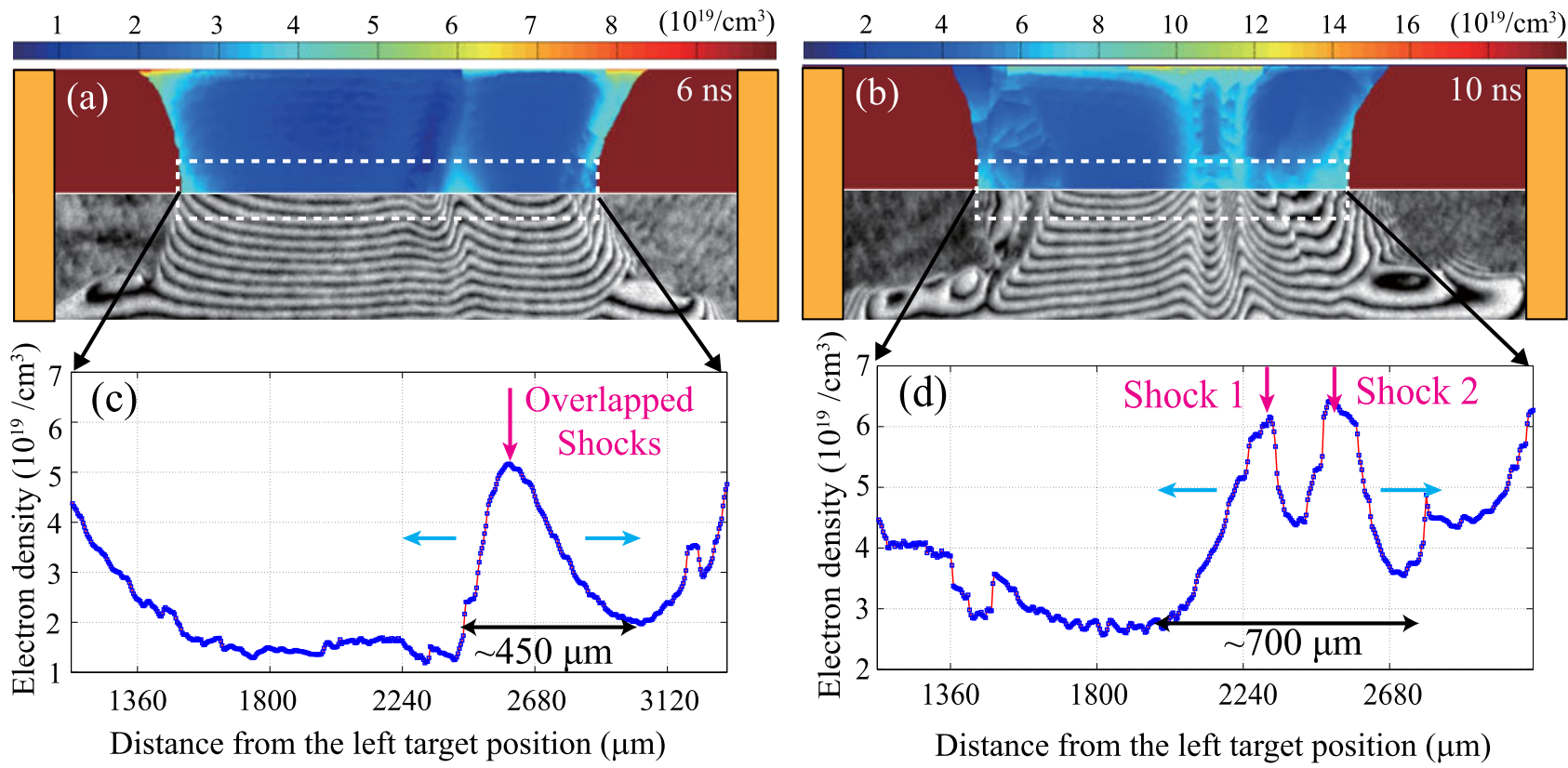

Figure 4. Collisionless shock formation and evolution in the symmetrical CPFs. (a) and (b) are the interferograms (lower) and electron density distributions (upper) obtained by the Abel inversion, taken at $6 \mathrm{~ns}$ and $10 \mathrm{~ns}$, respectively. (c) and (d) are the electron density profiles plotted along the flow direction ${ }^{\text {[20] }}$.

features of the Weibel instability can be observed in the shadowgraph. At $3.5 \mathrm{~ns}$, periodically distributing filaments parallel to flow direction localize at the midplane with a wavelength $(\lambda)$ of about $50-80 \mu \mathrm{m}$. The wavelength is almost consistent with the ion inertial scale, $d_{i}=c / \omega_{p i}=$ $c / \sqrt{4 \pi n_{i} \bar{Z}^{2} e^{2} / m_{i}} \approx 70-80 \mu \mathrm{m}$ (here the used flows density is $n_{e} \sim(2-3) \times 10^{19} \mathrm{~cm}^{-3}$ and $\left.\bar{Z} \sim 10\right)$, indicating that the instability is driven by the ions. The linear growth rate of the filamentation instability is approximated to be $\gamma \approx 0.2 v / \lambda=(2-3) \times 10^{9} \mathrm{~s}^{-1}$. The corresponding linear time of the filaments is about $2-3 \mathrm{~ns}(t=2 \pi / \gamma)$, after both flows meeting at the midplane. At $4.5 \mathrm{~ns}$, with the growth of instability, the filaments extend longer in the axial direction. The average space between adjacent filaments becomes $100-120 \mu \mathrm{m}$, indicating that the coalescence process between adjacent filaments occurs and the instability evolves from the linear mode into the nonlinear mode. At $6.5 \mathrm{~ns}$, the larger wavelength of order 150-180 $\mu \mathrm{m}$ and disordered filaments at the midplane reveal that the instability has fully evolved into the nonlinear mode. The experimental evolution of the Weibel instability is consistent with the theoretical prediction.

\subsection{Other applications of the CPFs}

The neutron yield in CPFs is an important tool to distinguish between collisionless and collisional effects. Neutrons generation in CPFs can originate from three sources: (i) the laser-induced fireballs from the target foils, (ii) the counterpropagating ions interaction with each other, and (iii) the scattering ions interaction with the ions from intra-flows. Here we carry out two neutron generation experiments for comparison ${ }^{[36]}$. One is for collisionless case (foil targets)high-velocity, low-density counter-streaming flows-and the other one is collisional case (K-shaped targets)-lowvelocity, high-density counter-streaming jets. The neutrons of $2.45 \mathrm{MeV}$ are produced by deuterium-deuterium (DD) nuclear reactions $(\mathrm{D}+\mathrm{D} \rightarrow 3 \mathrm{He}+n(2.45 \mathrm{MeV}))$. The typical signal of the $2.45 \mathrm{MeV}$ neutrons is shown in Figure 6. Since the nuclear cross section changes sharply at the limits of $2 \mathrm{MeV}$ of incident ions, it can be regarded as similar for both cases at the typically laser-produced flow velocity region $\left(10^{7}-10^{8} \mathrm{~cm} \cdot \mathrm{s}^{-1}\right)$. However, the density difference is very large. From the optical measurement, we can find that the flows are with density of $10^{19} \mathrm{~cm}^{-3}$, while the jets are with density larger than $10^{21} \mathrm{~cm}^{-3}$ (roughly $n_{\text {jet }} \sim$ $\left.100 n_{\text {flow }}\right)$. When we compare both cases, we can find that the neutron yields in collisional case $\left(10^{6}\right)^{[36]}$ is only $5-10$ times as much as that in collisionless case $\left((1-5) \times 10^{5}\right)^{[37]}$. One possible explanation is that the ions scattered by the self-generated electromagnetic field interaction with the ions from intra-flows will effectively enhance the neutron yields. This laser-produced CPF provides us not only a new tool to distinguish Coulomb collisions from the collisionless effects, i.e., random electromagnetic fields originated from instabilities, but also a platform for studying the nuclear astrophysics, such as the abundance of the ${ }^{26} \mathrm{Al}^{[38]}$ and ${ }^{6,7} \mathrm{Li}^{[39]}$. 

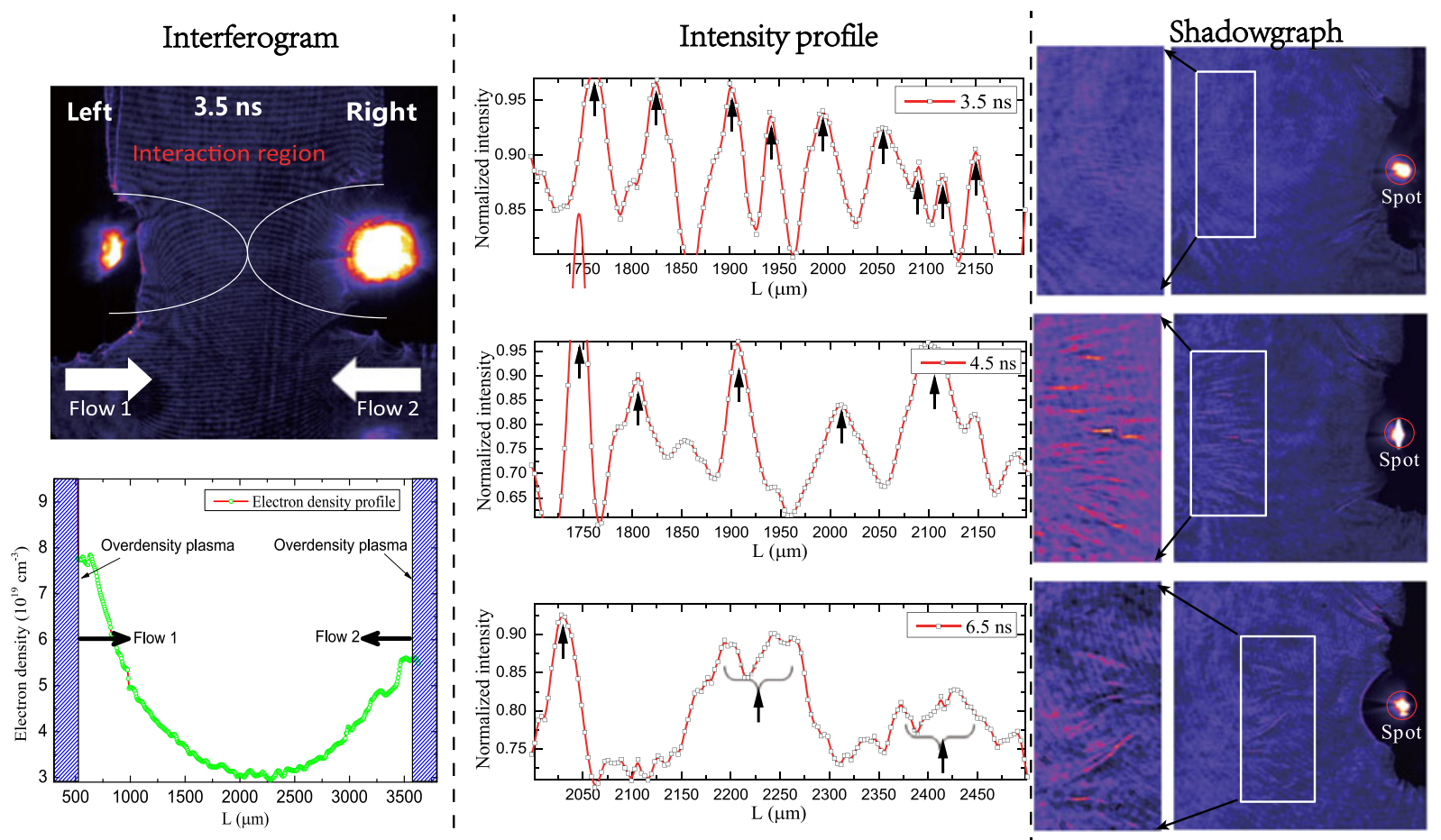

Figure 5. The evolution of the filamentation instability in CPFs. Interferogram with magnification of 2.5 times shows initial conditions of both flows at $3.5 \mathrm{~ns}$. A series of shadowgraph with larger magnification of 4 times is applied to measure the evolution of the Weibel instability. The intensity profile shows the typical features of the evolution of the filaments.

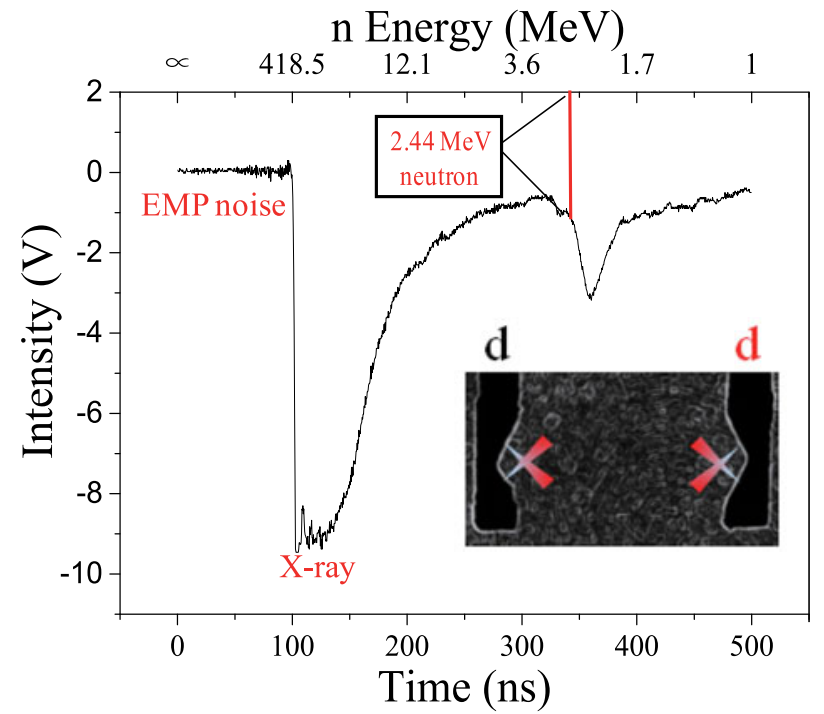

Figure 6. The observed neutron signals from D-D nuclear reaction in collisional case ${ }^{[36,40]}$

\section{Summary and outlook}

The study of astrophysical shock formation is important for us to understand the particle acceleration and cosmic rays generation. The laboratory experiments provide us a new opportunity to investigate the physical mechanism behind these scenarios. Our experimental results show that both electrostatic instability and filamentation instability can grow up in CPFs, but compete with each other. The selfgenerated bipolar electrostatic field from the electrostatic instability can support the shock formation and evolution. However, the magnetic field is too weak to excite the shock generation.

Considering the characteristic of the cosmic ray spectrum with power law, Weibel-mediated shock is a promising candidate via diffusive shock acceleration. Such important issues of the acceleration mechanism are not fully understood and still a big challenge. A stronger magnetic field is necessary to excite the Weibel-type shock. This higher magnetic field could be achieved by optimizing filamentation instability ${ }^{[34,35]}$ or laser-driven coils ${ }^{[41-43]}$ in future experiments.

\section{Acknowledgements}

The authors thank the staff of the SG-II laser facility for operating the laser and target area. This work is supported by the Science Challenge Project (No. TZ2016005), the National Basic Program of China (No. 2013CBA01501/03), the National Natural Science Foundation of China (Nos. $11503041,11522326,11622323$, and 11573040), the Strategic Priority Research Program of the Chinese Academy of 
Sciences (Nos. XDB16010200 and XDB07030300), and the Project Funded by China Postdoctoral Science Foundation (No. 2015M571124).

\section{References}

1. B. A. Remington, R. P. Drake, and D. D. Ryutov, Rev. Mod. Phys. 78, 755 (2006).

2. B. A. Remington, D. Arnett, R. P. Drake, and H. Takabe, Science 284, 1488 (1999).

3. J. E. Cross, B. Reville, and G. Gregori, Astrophys. J. 795, 59 (2014).

4. D. D. Ryutov, R. P. Drake, J. Kane, E. Liang, B. A. Remington, and W. M. Wood-Vasey, Astrophys. J. 518, 821 (1999).

5. J. J. Hester, Annu. Rev. Astron. Astrophys. 46, 127 (2008).

6. M. Yamada, R. Kulsrud, and H. Ji, Rev. Mod. Phys. 82, 602 (2010).

7. M. Yamada, J. Yoo, and C. E. Myers, Phys. Plasmas 23, 055402 (2016).

8. B. Reipurth and J. Bally, Annu. Rev. Astron. Astrophys. 39, 403 (2001).

9. B. Aya, Y. Ryo, U. Masaru, and K. Katsuji, Astrophys. J. 589, 526 (2003).

10. H. Takabe, T. N. Kato, Y. Sakawa, Y. Kuramitsu, T. Morita, T. Kadono, K. Shigemori, K. Otani, H. Nagatomo, T. Norimatsu, S. Dono, T. Endo, K. Miyanishi, T. Kimura, A. Shiroshita, N. Ozaki, R. Kodama, S. Fujioka, H. Nishimura, D. Salzman, B. Loupias, C. Gregory, M. Koenig, J. N. Waugh, N. C. Woolsey, D. Kato, Y.-T. Li, Q.-L. Dong, S.-J. Wang, Y. Zhang, J. Zhao, F.-L. Wang, H.-G. Wei, J.-R. Shi, G. Zhao, J.-Y. Zhang, T.-S. Wen, W.-H. Zhang, X. Hu, S.-Y. Liu, Y. K. Ding, L. Zhang, Y.J. Tang, B.-H. Zhang, Z.-J. Zheng, Z.-M. Sheng, and J. Zhang, Plamsa Phys. Control. Fusion 50, 124057 (2008).

11. M. V. Medvedev and A. Loeb, Astrophys. J. 526, 697 (1999).

12. P. Chang, A. Apitkovsky, and J. Arons, Astrophys. J. 674, 378 (2008).

13. F. A. Aharonian, A. G. Akhperjanian, K.-M. Aye, A. R. BazerBachi, M. Beilicke, W. Benbow, D. Berge, P. Berghaus, K. Bernlohr, O. Bolz, C. Boisson, C. Borgmeier, F. Breitling, A. M. Brown, J. Bussons Gordo, P. M. Chadwick, V. R. Chitnis, L.-M. Chounet, R. Cornils, L. Costamante, B. Degrange, A. Djannati-Ata, L. O'C. Drury, T. Ergin, P. Espigat, F. Feinstein, P. Fleury, G. Fontaine, S. Funk, Y. A. Gallant, B. Giebels, S. Gillessen, P. Goret, J. Guy, C. Hadjichristidis, M. Hauser, G. Heinzelmann, G. Henri, G. Hermann, J. A. Hinton, W. Hofmann, M. Holleran, D. Horns, O. C. de Jager, I. Jung, B. Khélifi, Nu. Komin, A. Konopelko, I. J. Latham, R. Le Gallou, M. Lemoine, A. Lemiere, N. Leroy, T. Lohse, A. Marcowith, C. Masterson, T. J. L. McComb, M. de Naurois, S. J. Nolan, A. Noutsos, K. J. Orford, J. L. Osborne, M. Ouchrif, M. Panter, G. Pelletier, S. Pita, M. Pohl, G. Puhlhofer, M. Punch, B. C. Raubenheimer, M. Raue, J. Raux, S. M. Rayner, I. Redondo, A. Reimer, O. Reimer, J. Ripken, M. Rivoal, L. Rob, L. Rolland, G. Rowell, V. Sahakian, L. Sauge, S. Schlenker, R. Schlickeiser, C. Schuster, U. Schwanke, M. Siewert, H. Sol, R. Steenkamp, C. Stegmann, J.-P. Tavernet, C. G. Théoret, M. Tluczykont, D. J. van der Walt, G. Vasileiadis, P. Vincent, B. Visser, H. J. Volk, and S. J. Wagner, Nature 432, 75 (2004).

14. Y. Uchiyama, F. A. Aharonian, T. Tanaka, T. Takahashi, and Y. Maeda, Nature 449, 576 (2007).

15. A. Spitkovsky, Astrophys. J. Lett. 682, L5 (2008).

16. C. Chenais-Popovics, P. Renaudin, O. Rancu, F. Gilleron, J.-C. Gauthier, O. Larroche, O. Peyrusse, M. Dirksmoller,
P. Sondhauss, T. Missalla, I. Uschmann, E. Forster, O. Renner, and E. Krousky, Phys. Plasmas 4, 190 (1997).

17. A. Ciardi, T. Vinci, J. Fuchs, B. Albertazzi, C. Riconda, H. Pépin, and O. Portugall, Phys. Rev. Lett. 110, 025002 (2013). 18. https://apod.nasa.gov/apod/ap090801.html.

19. J. Y. Zhong, J. Lin, Y. T. Li, X. Wang, Y. Li, K. Zhang, D. W. Yuan, Y. L. Ping, H. G. Wei, J. Q. Wang, L. N. Su, F. Li, B. Han, G. Q. Liao, C. L. Yin, Y. Fang, X. Yuan, C. Wang, J. R. Sun, G. Y. Liang, F. L. Wang, Y. K. Ding, X. T. He, J. Q. Zhu, Z. M. Sheng, G. Li, G. Zhao, and J. Zhang, Astrophys. J. Suppl. Ser. 225, 30 (2016).

20. D. Yuan, Y. Li, M. Liu, J. Y. Zhong, B. J. Zhu, Y. F. Li, H. G. Wei, B. Han, X. X. Pei, J. R. Zhao, F. Li, Z. Zhang, G. Y. Liang, F. L. Wang, S. M. Weng, Y. J. Li, S. E. Jiang, K. Du, Y. K. Ding, B. Q. Zhu, J. Q. Zhu, G. Zhao, and J. Zhang, Nat. Sci. Rep. 7, 42915 (2017).

21. D. Yuan and Y. Li, Chin. Phys. B 24, 015204 (2015).

22. D. W. Yuan, Y. T. Li, X. Liu, Y. Zhang, J. Y. Zhong, W. D. Zheng, Q. L. Dong, M. Chen, Y. Sakawa, T. Morita, Y. Kuramitsu, T. N. Kato, H. Takabe, Y.-J. Rhee, J. Q. Zhu, G. Zhao, and J. Zhang, High Energy Density Phys. 9, 239 (2013).

23. X. Liu, Y. T. Li, Y. Zhang, J. Y. Zhong, W. D. Zheng, Q. L. Dong, M. Chen, G. Zhao, Y. Sakawa, T. Morita, Y. Kuramitsu, T. N. Kato, L. M. Chen, X. Lu, J. L. Ma, W. M. Wang, Z. M. Sheng, H. Takabe, Y.-J. Rhee, Y. K. Ding, S. E. Jiang, S. Y. Liu, J. Q. Zhu, and J. Zhang, New J. Phys. 13, 093001 (2011).

24. D. Yuan, Y. Li, L. Su, G. Liao, C. Yin, B. Zhu, and J. Zhang, Sci. Chin. Phys. Mech. Astron. 56, 2381 (2013).

25. A. Stockem, F. Fiuza, A. Bret, R. A. Fonseca, and L. O. Silva, Nat. Sci. Rep. 4, 3934 (2014).

26. T. N. Kato and H. Takabe, Phys. Plasmas 17, 032114 (2010).

27. D. W. Forslund and C. R. Shonk, Phys. Rev. Lett. 25, 1699 (1970).

28. E. S. Weibel, Phys. Rev. Lett. 2, 83 (1959).

29. W. Fox, G. Fiksel, A. Bhattacharjee, P.-Y. Chang, K. Germaschewski, S. X. Hu, and P. M. Nilson, Phys. Rev. Lett. 111, 225002 (2013).

30. T. Morita, Y. Sakawa, Y. Kuramitsu, S. Dono, H. Aoki, H. Tanji, T. N. Kato, Y. T. Li, Y. Zhang, X. Liu, J. Y. Zhong, H. Takabe, and J. Zhang, Phys. Plasmas 17, 122702 (2010).

31. Y. Sakawa, T. Morita, Y. Kuramitsu, and H. Takabe, Adv. Phys. 3, 425 (2016).

32. H.-S. Park, D. D. Ryutov, J. S. Ross, N. L. Kugland, S. H. Glenzer, C. Plechaty, S. M. Pollaine, B. A. Remington, A. Spitkovsky, L. Gargate, G. Gregori, A. Bell, C. Murphy, Y. Sakawa, Y. Kuramitsu, T. Morita, H. Takabe, D. H. Froula, G. Fiksel, F. Miniati, M. Koenig, A. Ravasio, A. Pelka, E. Liang, N. Woolsey, C. C. Kuranz, R. P. Drake, and M. J. Grosskopf, High Energy Density Phys. 8, 38 (2012).

33. J. S. Ross, D. P. Higginson, D. Ryutov, F. Fiuza, R. Hatarik, C. M. Huntington, D. H. Kalantar, A. Link, B. B. Pollock, B. A. Remington, H. G. Rinderknecht, G. F. Swadling, D. P. Turnbull, S. Weber, S. Wilks, D. H. Froula, M. J. Rosenberg, T. Morita, Y. Sakawa, H. Takabe, R. P. Drake, C. Kuranz, G. Gregori, J. Meinecke, M. C. Levy, M. Koenig, A. Spitkovsky, R. D. Petrasso, C. K. Li, H. Sio, B. Lahmann, A. B. Zylstra, and H.-S. Park, Phys. Rev. Lett. 118, 185003 (2017).

34. H.-S. Park, C. M. Huntington, F. Fiuza, R. P. Drake, D. H. Froula, G. Gregori, M. Koenig, N. L. Kugland, C. C. Kuranz, D. Q. Lamb, M. C. Levy, C. K. Li, J. Meinecke, T. Morita, R. D. Petrasso, B. B. Pollock, B. A. Remington, H. G. Rinderknecht, M. Rosenberg, J. S. Ross, D. D. Ryutov, Y. Sakawa, A. Spitkovsky, H. Takabe, D. P. Turnbull, P. Tzeferacos, S. V. Weber, and A. B. Zylstra, Phys. Plasmas 22, 056311 (2015). 
35. C. M. Huntington, M. J.-E. Manuel, J. S. Ross, S. C. Wilks, F. Fiuza, H. G. Rinderknecht, H.-S. Park, G. Gregori, D. P. Higginson, J. Park, B. B. Pollock, B. A. Remington, D. D. Ryutov, C. Ruyer, Y. Sakawa, H. Sio, A. Spitkovsky, G. F. Swadling, H. Takabe, and A. B. Zylstra, Phys. Plasmas 24, 041410 (2017).

36. J. R. Zhao, L. M. Chen, Y. T. Li, C. B. Fu, Y. J. Rhee, F. Li, B. J. Zhu, Y. F. Li, G. Q. Liao, K. Zhang, B. Han, C. Liu, K. Huang, Y. Ma, Y. F. Li, J. Xiong, X. G. Huang, S. Z. Fu, J. Q. Zhu, G. Zhao, and J. Zhang, Rev. Sci. Instrum. 86, 063505 (2015).

37. X. Zhang, J. Zhao, D. Yuan, C. Fu, J. Bao, L. Chen, J. He, L. Hou, L. Li, Y. Li, Y. Li, G. Liao, Y. Rhee, Y. Sun, S. Xu, G. Zhao, B. Zhu, J. Zhu, Z. Zhang, and J. Zhang, Phys. Rev. C 96, 055801 (2017).

38. N. Prantzos and R. Diehl, Phys. Rep. 267, 1 (1996).

39. R. H. Cyburt, B. D. Fields, K. A. Olive, and T. H. Yeh, Rev. Mod. Phys. 88, 015004 (2016).
40. J. R. Zhao, X. P. Zhang, D. W. Yuan, Y. T. Li, D. Z. Li, Y. J. Rhee, Z. Zhang, F. Li, B. J. Zhu, Y. F. Li, B. Han, C. Liu, Y. Ma, Y. F. Li, M. Z. Tao, M. H. Li, X. Guo, X. G. Huang, S. Z. Fu, J. Q. Zhu, G. Zhao, L. M. Chen, C. B. Fu, and J. Zhang, Nat. Sci. Rep. 6, 27363 (2016).

41. S. Fujioka, Z. Zhang, K. Ishihara, K. Shigemori, Y. Hironaka, T. Johzaki, A. Sunahara, N. Yamamoto, H. Nakashima, T. Watanabe, H. Shiraga, H. Nishimura, and H. Azechi, Nat. Sci. Rep. 3, 1170 (2013).

42. G. Q. Liao, Y. F. Li, F. Li, M. C. Li, X. Wang, Z. Zhang, S. K. He, W. W. Wang, F. Lu, F. Q. Zhang, L. Yang, K. N. Zhou, N. Xie, W. Hong, Y. Q. Gu, Z. Q. Zhao, B. H. Zhang, and J. Zhang, Matter \& Radiation at Extremes 1, 187 (2016).

43. B. J. Zhu, Y. T. Li, D. W. Yuan, Y. F. Li, F. Li, G. Q. Liao, J. R. Zhao, J. Y. Zhong, F. B. Xue, S. K. He, W. W. Wang, F. Lu, F. Q. Zhang, L. Yang, K. N. Zhou, N. Xie, W. Hong, H. G. Wei, K. Zhang, B. Han, X. X. Pei, C. Liu, Z. Zhang, W. M. Wang, J. Q. Zhu, Y. Q. Gu, Z. Q. Zhao, B. H. Zhang, G. Zhao, and J. Zhang, Appl. Phys. Lett. 107, 261903 (2015). 